NEUROLOGICAL PICTURE

\title{
Peripheral axonal motor degeneration after spinal cord infarct
}

A 62 year old male hypertensive patient with known diabetes mellitus and diffuse arteriopathy was admitted for cardiac failure. On hospital day 2, septic shock due to an unidentified micro-organism, encephalopathy, and flaccid paraplegia developed. On day 4, dry gangrene of all toes and progressive coma appeared. He died on hospital day 22 of multiorgan failure.

Nerve conduction studies carried out on day 9 showed progressive loss of compound muscle action potential (CMAP) amplitude of the right and left abductor hallucis muscle with sequential stimulation of the tibial nerve (fig 1 (A): right side, superimposed mode. (B), left side, raster mode, with stimulation of the nerve at the ankle (first CMAP), and at 3, 6, and $9 \mathrm{~cm}$ proximal to the ankle (second to fourth CMAPs), and at the popliteal fossa (fifth CMAP)). Sural nerve action potentials were within the lower range of the normal value $(5 \mu \mathrm{V})$. Motor and sensory nerve study indices in the right arm were normal.

Postmortem examination showed a complete transverse ischaemic infarction of the spinal cord (central insert fig1; haematoxylin and eosin; bar $=2.5 \mathrm{~mm}$ ) extending from thoracic level 8 to the caudal end of the conus medullaris, with anterograde degeneration of nerve fibres in the anterior roots (fig $\mathrm{C}$ (longitudinal) and $\mathrm{E}$ (transverse); avidin-biotinperoxidase technique with monoclonal antibodies to neurofilament protein; bar $=20 \mu \mathrm{m}$ ) and preservation of nerve fibres in the dorsal roots (fig1 D and F, same as in $\mathrm{C}$ and $\mathrm{E}$ ), as well as an acute thrombosis of the femoral arteries.
The progressive inexcitablity of the terminals of the sciatic nerves when moving the electric stimulation proximally from the motor points illustrates the ongoing process of wallerian degeneration. ${ }^{1-3}$ Electrophysiologically, this phenomenon can only be demonstrated after a latent period proportional to the length of the nerve distal to the site of injury. In the distal lower limb muscles, complete wallerian degeneration is achieved between 2 to 4 weeks after very proximal lesions. ${ }^{3}$ This progressive distal to proximal CMAP amplitude loss differs from conduction block, where the drop in amplitude is stepwise over short segments. Postmortem examination confirmed subacute wallerian degeneration in motor roots after destruction of the motor neurons in the anterior horn. The ischaemic cord infarction probably resulted from embolic infarction of the artery of Adamkiewicz. The ascending sensory nerve roots and nerves were not affected (fig $1 \mathrm{D}$ and $\mathrm{E}$ ) as their perikaryia, lying outside the spinal cord, were spared.

P MICHEL

J MIKLOSSY

T KUNTZER

Service de neurologie and Institut Universitaire de Pathologie, Centre

Hospitalier Universitaire Vaudois, 1011 Lausanne, Switzerland

Thierry.kuntzer@chuv.hospvd.ch

1 Erlanger J, Schoepfle GM. A study of nerve degeneration and regeneration. f Physiol 1946;147:550-81.

2 Gilliatt RW. Physical injury to peripheral nerves. Physiologic and electrodiagnostic aspects. Mayo Clin Proc 1981;56:361-70.

3 Brown WF. Negative symptom and signs of peripheral nerve disease. In: WF Brown, CF Bolton, eds. Clinical electromyography. Boston: ButterworthHeinemann, 1993:93-116.

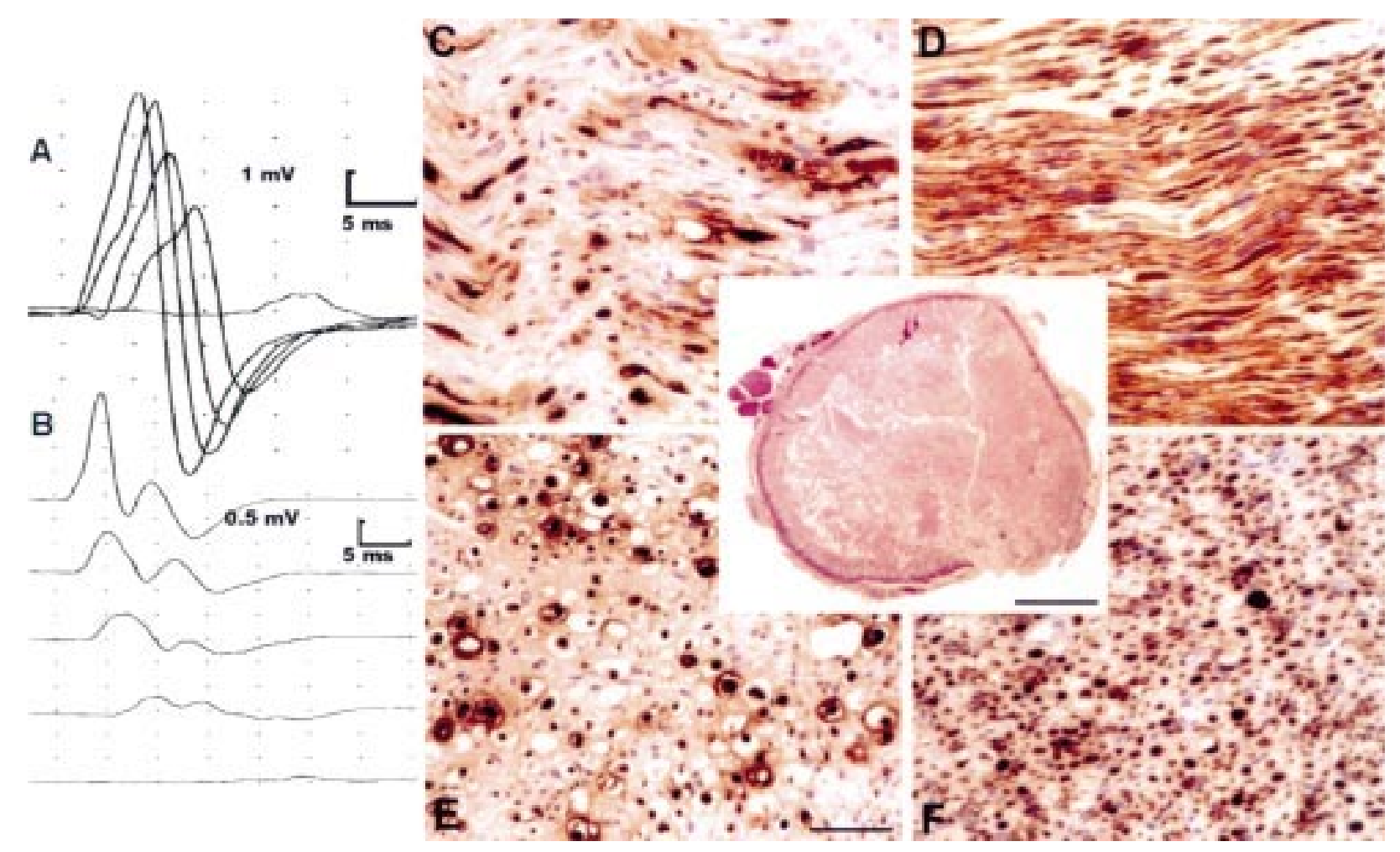

Figure 1 